\section{Supraconductivity and the Hall Effect}

IN connexion with the recent discovery of several new supraconductors ${ }^{1}$, a re-investigation of zinc was undertaken, in development of the previous work of this laboratory ${ }^{2}$. In polycrystalline zinc, the $R \sigma$ value is so big that the recent discovery of supraconductivity in this metal should be considered as an exception to our previously established rule. But zinc crystallises in the hexagonal system and possesses a very definite anisotropy of physical properties. For this reason we decided to investigate monocrystalline zinc. Two kinds of plates of it were prepared : in one, the main axis lies in the plane of the plate, the primary current being parallel to the direction of the axis; in the other, the plane of the plate is perpendicular to the main axis. For both orientations the Hall coefficients have been determined, the corresponding conductivities parallel to $\left(\sigma_{1}\right)$ and perpendicular to $\left(\sigma_{2}\right)$ the axis, being taken from the tables. We obtained the following results :

$$
\begin{aligned}
& \mid R \sigma_{1}=14 \\
& |R \sigma|_{2}=300 .
\end{aligned}
$$

Thus it is seen that the violation of our rule in this case is only an apparent one. Zinc seems to belong to the group of supraconductors in one particular crystallographic direction only, and it supports strongly our rule.

It should be very important to determine the supraconductivity phenomena in different crystallographic directions. From the experimental point of view, such an investigation is, however, likely to moet very considerable difficulties, especially concerning the interpretation of the experimental results thus obtained.

\section{Physical Technical Institute of the Ural, \\ Sosnovka 2, Leningrad, 21. June 4.} ${ }^{1}$ W. Meissner, $Z$. Phys., 87, $206 ; 1933$ - Supraconductivity of
vanadium. W. H. Keesom, Proc. Amsterdam, 36, 381; 1933-Supraconductivity of aluminium. W. H. Keesom, Physica, 1, 123 ; 1934Supraconductivity of zine.

Kikoin and Lasarew, NATURE, 129, 57, Jan. 9, 1932. Phys. Z. Sowjetunion, 3, 351; 1933. Dorfman, Metallwirtschaft, 12, 221 1933. Lasarew, Phys. Z. Sowjetunion, 4, 567; 1933

\section{Nature of a Magnetic Field}

IN a paper read before Section $\mathbf{A}$ of the British Association last year, I gave some account of experiments made by Dr. Norgrove and myself on cylindrical magnets and solenoids spinning about their axes. These experiments forced us towards the conclusion that even in the strongest permanent magnet there was no evidence of any attachment between the metal and the system of tubes of induction to which it is supposed to give rise. All our tests then and since have only confirmed Faraday's words as to the "singular independence of the magnetism and the bar in which it resides".

It has occurred to me recently that, apart altogether from the tests referred to above, accepted theory also supports our view as to the stationary magnetic flux. Zeleny and Page ${ }^{1}$ have shown that no current circuit, even if it passes in part through the magnet, can exert a torque about the axis of symmetry of a symmetrically magnetised cylinder. This is entirely in accord with our experiments. It follows that the torque exerted on the magnet by the current can only be due to the interaction between the field and current within the magnet. If this be admitted, the question as to whether the tubes of induction are attached to the metal of the magnet is at once settled. Consider a horizontal section of a vertical cylindrical magnet through which the flux of induction $\phi$ is upwards. If now current $i$ pass radially inwards at the equator, the torque is $i \varphi / 2 \pi$, and the magnet tends to turn in the positive direction viewed from above. To produce this couple, if one of the components $i$ or $\varphi$ is assumed to be attached to the particles of the magnet, the other must be independent thereof. But the direction of the twist shows us which is which. For if the tubes of induction were attached to the molecules of the metal, while the current sheet remained independent and stationary, the motion of the magnet would be in a direction opposite to that which is found by experiment to exist. The conclusion, therefore, is unavoidable that the magnetic field is the independent and stationary quantity, the drag on the metal being due to the deflection of the moving electrons forming the current which must in their turn, either by collisions or by attractions, act upon the atoms of the metal.

Two further results follow. First, if a current is led into and away from a rotating bar of metal of any kind, more power will be needed to cause the rotation when the current is flowing than when it is switched off. Second, in an electric circuit made up of stationary conductors and a spinning magnet, the generation of the E.M.F. takes place in the magnet itself, and not in the stationary conductors.

The University,

WILLIAM Cramp.

Edgbaston, Birmingham, 15. July 5.

${ }^{1}$ Phys. Rev., Ser. 2, 24, 544.

\section{Measurement of Ultra-Violet Light}

Is measuring the amount of ultra-violet light reaching the earth from sun and sky, it is customary to expose the acetone methylene blue tube in the vertical position. Since more ultra-violet light is received from the sun itself than from the whole of the rest of the sky put together, the vertical position seems unsuitable, for the amount of ultra-violet light received by the contents of the tube will be too low during the time of day and year when the sun is most nearly overhead. The horizontal position has occasionally been used, but this is open to the opposite criticism.

The Oxford University Expedition has recently been measuring the ultra-violet light in the New Hebrides, in the tropical Pacific. If we had used the vertical position for the tube, then, on the two days of the year on which the sun passed directly overhead, the tube would have received practically no ultra-violet light from the sun at the very time (midday) when the irradiation was likely to be at its greatest. We therefore always exposed both the quartz tube and the control glass tube on a simple wooden stand (Fig. 1) kindly made for us by my brother, Mr. S. J. Baker. This stand was set up so that the ends of the tubes always pointed north and south, and their inclination was changed each month so that the rays of the sun always fell approximately at right angles upon them. (Weekly changes could 